\title{
MULTIPARAMETER ROOT VECTORS
}

\author{
by PAUL BINDING*
}

(Received 27th February 1987, revised June 1987)

\section{Preliminaries}

The concept of "root vectors" is investigated for a class of multiparameter eigenvalue problems

$$
W_{m}(\lambda) x_{m}=0 \neq x_{m}, \quad m=1, \ldots, k
$$

where $W_{m}(\lambda)=T_{m}-\sum_{n=1}^{k} \lambda_{n} V_{m n}$ operate in Hilbert spaces $H_{m}$ and $\lambda \in \mathbb{C}^{k}$. Previous work on this "uniformly elliptic" class has demonstrated completeness of the decomposable tensors $x_{1} \otimes \cdots \otimes x_{k}$ in a subspace $G$ of finite codimension in $H=H_{1} \otimes \cdots \otimes H_{k}$, but questions remain about extending this to a basis of $H$. In this work, bases of elements $y_{m}$, in general nondecomposable but satisfying recursive equations of the type $W_{m}(\lambda) y_{m}$ $=\sum_{n=1}^{k} V_{m n} z_{m n}$, are constructed for the "root subspaces" corresponding to $\lambda \in \mathbb{R}^{k}$.

\section{Introduction}

Let $T_{m}, V_{m n}$ be self-adjoint operators in Hilbert spaces $H_{m}, T_{m}$ being bounded below with compact resolvent, and $V_{m n}$ being bounded, for $1 \leqq m, n \leqq k$. We are interested in a spectral decomposition of the Hilbert Space tensor product $H=H_{1} \otimes \cdots \otimes H_{k}$ by the eigenvalue problem $(*)$ of Section $\mathbf{0}$.

Let us begin with the case $k=1$, when (*) becomes, with subscripts suppressed,

$$
W(\lambda) x=0, W(\lambda)=T-\lambda V .
$$

Despite the self-adjointness assumptions, $\lambda$ need not be real and the eigenvectors $x$ need not be complete in $H$. Under a suitable nondegeneracy condition (e.g. if $V$ is $1-1$ ), it can be shown [6] that the span $G$ of the eigenvectors has a finite dimensional complement $F$ which is in turn spanned by elements $x^{j}$ satisfying equations of the form

$$
W(\lambda) x^{j}=V x^{j-1}, \quad j=0, \ldots, l-1
$$

where $x^{-1}=0$. Evidently this is equivalent to the Jordan chain condition

$$
(\Gamma-\lambda) x^{j}=x^{j-1}
$$

*Research supported by a Canadian NSERC Operating Grant. 
where $\Gamma=V^{-1} T$ so $x^{j-1} \in N(\Gamma-\lambda I)^{j}$. The $x^{j}$ are called root vectors and

$$
N(\Gamma-\lambda I)^{d}
$$

$d=\operatorname{dim} F$, is called the root subspace associated with $\lambda$.

For $k>1$, there seems to be no analogue in the literature, although various authors have addressed the problem. Atkinson [1] raises the question of how to define root vectors for $k>1$ and gives one answer as follows, at least in finite dimensions [2, Chapter 6]. With $\dagger$ denoting induced operators in $H$ (e.g. $V_{11}^{\dagger}=V_{11} \otimes I_{2} \otimes \cdots \otimes I_{k}$ ), we set

$$
\Delta_{0}=\operatorname{det}\left[V_{m n}^{\dagger}\right]
$$

which is well defined since the elements of different rows commute. Then $\Delta_{n}$ is defined as the determinant in (1.3) but with column $n$ replaced by $\left[T_{1}^{\dagger}, \ldots, T_{k}^{\dagger}\right]^{T \text {. Under a }}$ suitable nondegeneracy condition (e.g. if $\Delta_{0}$ is $1-1$ ) the operators $\Gamma_{n}=\Delta_{0}^{-1} \Delta_{n}$ commute for $n=1, \ldots, k$ and thus $H$ admits a decomposition into joint root subspaces of the form

$$
J(\lambda)=\bigcap_{n=1}^{k} N\left(\left(\Gamma_{n}-\lambda_{n} I\right)^{v}\right)
$$

where $v \leqq \operatorname{dim} H$, cf. (1.2).

This leads to a rather complicated definition of root vectors, since an element of (1.4) will in general belong to different Jordan chains for each $\Gamma_{n}$, cf. (1.1), and moreover such chains are not defined directly in terms of the data in (*). This is particularly important when $\operatorname{dim} H=\infty$, since the construction and commutativity of the $\Gamma_{n}$ are then by no means obvious. In a more general situation, Isaev [10] has addressed the relation between elements of (1.4) and equations of the form

$$
W_{m}(\lambda)^{\dagger} x=\sum_{n=1}^{k} V_{m n}^{\dagger} z_{n}
$$

in $H$, but concludes that the topic "faces essential difficulties". Gadzhiev [9] has shown the relevance of tensors, formed from generalized chains satisfying equations of the form

$$
W_{m}(\lambda) x_{m}^{j}=\sum_{n=1}^{k} V_{m n} x_{m}^{j-1}
$$

in $H_{m}$, to systems of differential equations with multiple time scales. Our root vectors will be formed from a generalisation of (1.6) and will satisfy (1.5), for a class of problems obeying a "definiteness condition" defined below.

The simplest of many definiteness conditions in the literature on (*) is uniform right definiteness (URD) where $\Delta_{0} \gg 0$, i.e. has a positive definite bounded inverse, on $H$. It is known that URD holds if $\left(u, \Delta_{0} u\right)$ has a positive lower bound for unit decomposable tensors, giving a condition expressible directly in terms of the data in (*). Also URD 
implies that each $\lambda \in \mathbb{R}^{k}$ in (*), that each exponent $v$ in (1.4) may be taken as unity, and that $\bigcap_{n=1}^{k} N\left(\Gamma_{n}-\lambda_{n} I\right)$ is spanned by eigentensors, i.e. elements

$$
x^{\otimes}=x_{1} \otimes \cdots \otimes x_{k},
$$

where $x_{m}$ satisfy (*). References for these facts are $[2,3,11]$.

Another important definiteness condition, with application to various separation of variables problems, is uniform ellipticity (UE) where, instead of $\Delta_{0}$, the cofactors of $\Delta_{0}$, labelled $\Delta_{0 m n}, \gg 0$ on $H$. For various equivalent conditions, see [3] where UE is labelled $L D_{\delta}$-again UE may be checked directly in terms of the data in (*). Under a suitable nondegeneracy condition, e.g. if $\Delta_{0}$ is $1-1$, the span $G$ of the eigentensors (1.7) has a finite dimensional complement $F$ which is in turn spanned by joint root subspaces (1.4). This is an easy consequence of [7, Lemma 4.2] and will be demonstrated in Section 2. In the special case when each $T_{m} \gg 0$ on $H_{m}$, known as uniform left definiteness (ULD), each exponent $v$ may be taken as unity in (1.4), so the eigentensors (1.7) span $H$, as for URD, cf. $[4,13]$. Actually this holds under the weaker condition of UE and $\Delta_{n} \gg 0$ for some $n$. This will be seen in Section 4, but has already been observed for the case of $k$ $=2$ Sturm-Liouville equations $(*)$ in [8, Theorem 4.3].

This work of Faierman makes important contributions both to the completeness of eigentensors in $G$ (cf. the discussion in [7, Section 1]) and to the nature of root vectors required to span $F$. In the case when $\Delta_{2} \geqq 0,[8$, Theorem 5.5] gives a basis for $F$ in terms of the data in (*), and we shall discuss this further in Section 4 , noting here that in general $\lambda$ has real components and $\nu=2$ suffices in (1.4), cf. [7, Theorem 5.4]. When $\Delta_{2}$ is indefinite, [8, Theorem 9.2] gives a basis of $N\left(\Gamma_{2}-\lambda_{2} I\right)$ and in Section 3 we shall give an extension of this to general $v, k$ and $\lambda \in \mathbb{R}^{k}$, for our abstract formulation. While our methods also have a bearing on $\lambda \notin \mathbb{R}^{k}$, they do not cover all possibilities, and we hope to discuss the nonreal situation separately. In Section 2 we discuss the nondefective case $(v=1)$ and we embed (*) in a parametric family which is almost always non-defective. In Section 3 we use analytic perturbation theory, cf. [5], to discuss the defective case by a limiting process, and we connect our work with (1.5) and (1.6). Section 4 is devoted to remarks on determination of the root vectors, on Jordan structure of the $\Gamma_{n}$ and on the case where one of the $\Delta_{n} \geqq 0$. We conclude with a numerical example.

\section{The nondefective case}

We shall need certain constructions from [7]. Self adjoint operators $T_{m}$ and $V_{m n}$ are induced in $H$ by $T_{m}$ and $V_{m n}$, and $\Delta_{0}$ is defined by (1.3), with $\Delta_{0 m n}$ as the $(m, n)$ cofactor of this determinant. We assume (i) UE, i.e. each $\Delta_{0 m n} \gg 0$, and (ii) $\Delta_{0}$ is $1-1$. Then each operator

$$
\sum_{m=1}^{k} \Delta_{0 m n} T_{m}^{\dagger}
$$

has a self-adjoint closure in $H$, denoted by $\Delta_{n}$. If, for fixed $m$, we replace $V_{m n}$ by $\delta_{m n} I_{m}$ $\left(I_{m}=\right.$ identity on $H_{m}$ ) for $n=1, \ldots, k$ then $\Delta_{l}$ is replaced by a "cofactor" operator which 
we denote by $\Delta_{l m m}$. As in [7, Theorem 2.5] we may assume (by translating the $\lambda$ origin if necessary) that each $\Delta_{n}$ is bounded below with compact inverse, and we define

$$
B_{n}=\Delta_{n}^{-1} \Delta_{0}, \Gamma_{n}=\Delta_{0}^{-1} \Delta_{n}, \quad n=1, \ldots, k .
$$

Theorem 2.1. $H$ is the closure of $F \dot{+} G$ where $F$ is a finite dimensional direct sum of joint root subspaces (1.4) and $G$ is a linear span of eigentensors (1.7).

Proof. In [7, Lemma 4.2] it is shown that $D\left(\left|\Delta_{1}\right|^{1 / 2}\right)$ is the closure, in a norm stronger than that in $H$, of $F \dot{+} G$ say where $\operatorname{dim} F<\infty$ and the eigentensors span $G . F$ is a direct sum of joint root subspaces for the $B_{n}$, and an easy computation shows that $N\left(B_{n}-\lambda_{n}^{-1} I\right)^{v}=N\left(\Gamma_{n}-\lambda_{n} I\right)^{v}$, so the result follows from density of $D\left(\left|\Delta_{1}\right|^{1 / 2}\right)$ in $H$.

From now on we shall concentrate on the subspace $F$. If $v=1$ suffices in (1.4) for a fixed $\lambda$ then we say that $\lambda$ is nondefective. If each eigenvalue $\lambda$ is nondefective then (*) is nondefective.

Corollary 2.2. If $(*)$ is nondefective then $F$, and hence $H$, is spanned by eigentensors.

Proof. By [7, Theorem 3.2] the equations

$$
\left(B_{n}-\lambda_{n}^{-1}\right) x=0, \quad n=1, \ldots, k
$$

are equivalent to

$$
W_{m}(\lambda)^{\dagger} x=0, \quad m=1, \ldots, k
$$

and hence to

$$
x \in \bigotimes_{m=1}^{k} N\left(W_{m}(\lambda)\right)
$$

It suffices therefore to construct an eigentensor basis out of arbitrary basis elements $x_{m} \in N\left(W_{m}(\lambda)\right)$, for each $\lambda$ corresponding to a joint root subspace in $F$.

The basis of our subsequent analysis in an embedding with $T_{k}$ replaced by $T_{k}+\mu I_{k}$, $\mu \in \mathbb{R}$. Then $\Delta_{0}$ remains unchanged but $\Delta_{n}$ is replaced by $\Delta_{n}+\mu \Delta_{0 k n}$.

Theorem 2.3. The set of $\mu$ values for which (*) is defective has no finite accumulation.

Proof. Eliminating all but $\lambda_{n}$ from (*) we obtain

$$
\left(\Delta_{n}+\mu \Delta_{0 k n}-\lambda_{n} \Delta_{0}\right) x^{\otimes}=0
$$

i.e.

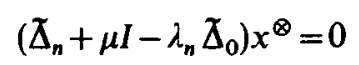


where $\tilde{\Delta}_{j}=\Delta_{0 k n}^{-1} \Delta_{j}(j=0, n)$ are self-adjoint in $H_{0 k n}$. Here $H_{0 k n}$ denotes $H$ with inner product given by $(x, y)_{0 k n}=\left(x, \Delta_{0 k n} y\right)$. We shall prove that the set of $\mu$ values for which (2.1) is defective (as a problem in $\lambda_{n}$ ) has no finite accumulation for any fixed $n$, and hence for all $n$. For other values of $\mu, \lambda_{n}$ will be a nondefective eigenvalue of

$$
\Gamma_{n}(\mu):=\triangle_{0}^{-1}\left(\triangle_{n}+\mu I\right)
$$

and so $v=1$ will suffice in (1.4).

For large real $\mu, \delta_{n}+\mu I \gg 0$ and hence has a positive square root $S$. Thus $\Gamma_{n}(\mu)^{-1}$ $=S^{-2} \bar{\Delta}_{0}$ is compact symmetric in $D(S)$ with inner product given by $[x, y]=(S x, S y)$. It follows that all eigenvalues of $\Gamma_{n}(\mu)^{-1}$, and hence of $\Gamma_{n}(\mu)$, are nondefective. Moreover $\Gamma_{n}(\mu)$ is holomorphic in $\mu$ [5, Lemma 3.2] and we then conclude that the eigennilpotents for $\Gamma_{n}(\mu)$ vanish for large real $\mu$, and hence for all $\mu$ [13, Theorem VII.1.8].

Suppose $\lambda_{j}$ is a defective (i.e. nonsemisimple) eigenvalue for $\Gamma_{n}\left(\mu_{j}\right)$, with $\mu_{j} \rightarrow \mu_{0}$ as $j \rightarrow \infty$. Without loss of generality we may assume $\lambda_{j} \rightarrow \lambda_{0}$ by virtue of [5, Theorem 3.7]. Appealing to [13, Section VII.1.3] we may separate $\sigma\left(\Gamma_{n}\left(\mu_{j}\right)\right)$ by means of a small contour in $\mathbb{C}$ encircling $\lambda_{0}$. This leads to a finite dimensional problem with a defective eigenvalue for each sufficiently large $\mu_{j}$. From the previous paragraph, such $\mu_{j}$ are exceptional in the sense of $\left[13\right.$, p. 64], and their accumulation at $\mu_{0}$ is therefore a contradiction.

In summary, we find that the eigentensors are complete in $H$ for almost all $\mu$. On the other hand $\mu=0$ may still yield a defective problem, and we turn next to this case.

\section{The defective case}

We fix our attention on a defective $\lambda^{*} \in \mathbb{R}^{k}$ corresponding to $\mu=0$. For notational ease, we shall consider first the simple case, when $\widetilde{\Delta}_{k}-\lambda_{k}^{*} \widetilde{\Delta}_{0}$, which is a self-adjoint operator on $H_{0 k k}$ in the notation of (2.1), has nullity one. By [13, Theorem VII.3.9] there exist real $\mu\left(\lambda_{k}\right)$, and $x\left(\lambda_{k}\right)$ of unit norm in $H_{0 k k}$, holomorphic at $\lambda_{k}^{*}$, such that

$$
N\left(\lambda_{k}\right):=N\left(\bar{\Delta}_{k}+\mu\left(\lambda_{k}\right) I-\lambda_{k} \bar{\Delta}_{0}\right)=N\left(\Gamma_{k}\left(\mu\left(\lambda_{k}\right)\right)-\lambda_{k} l\right)
$$

is spanned by $x\left(\lambda_{k}\right)$, in the notation of (2.2). Moreover the $\Gamma_{n}\left(\mu\left(\lambda_{k}\right)\right)$ commute for each $\lambda_{k}$ [7, Theorem 3.1], so they have eigenvalues $\lambda_{n}\left(\lambda_{k}\right)$ and a common eigenvector $x\left(\lambda_{k}\right)$. By [7, Theorem 3.2], $x\left(\lambda_{k}\right)$ is a decomposable tensor $x^{\otimes}\left(\lambda_{k}\right)$ say, where

$$
\begin{gathered}
W_{m}\left(\lambda\left(\lambda_{k}\right)\right) x_{m}\left(\lambda_{k}\right)=0, \quad 1 \leqq m<k \\
W_{k}\left(\lambda\left(\lambda_{k}\right)\right) x_{k}\left(\lambda_{k}\right)=-\mu\left(\lambda_{k}\right) x_{k}\left(\lambda_{k}\right) .
\end{gathered}
$$

Eliminating all but $\lambda_{j}$ and $\lambda_{k}$ from the first $k-1$ equations (3.2), we obtain

$$
\left(\Delta_{j k k}-\lambda_{j}\left(\lambda_{k}\right) \Delta_{0 k k}+\lambda_{k} \Delta_{0 k j}\right) x^{\otimes}\left(\lambda_{k}\right)=0
$$


in terms of the cofactor operators introduced in the first paragraph of Section 2 . Operating by $\Delta_{0 k k}^{-1}$, we derive an equation analogous to (2.1), viz.

$$
\left(\bar{\Delta}_{j k k}-\lambda_{j}\left(\lambda_{k}\right) I+\lambda_{k} \bar{\Delta}_{0 k j}\right) x^{\otimes}\left(\lambda_{k}\right)=0,
$$

involving self-adjoint operators on $H_{0 k k}$. It follows that $N\left(\lambda_{k}\right)$ is invariant for $\tilde{\Delta}_{j k k}$ $+\lambda_{k} X_{0 k j}$. Applying $\left[13\right.$, p. 386] to the $\left(H_{0 k k}\right)$ orthoprojector $P\left(\lambda_{k}\right)$ onto $N\left(\lambda_{k}\right)$, we construct an $\left(H_{0 k k}\right)$ unitary operator $U\left(\lambda_{k}\right)$, holomorphic at $\lambda_{k}^{*}$, such that

$$
U\left(\lambda_{k}\right)^{-1} P\left(\lambda_{k}^{*}\right) U\left(\lambda_{k}\right)=P\left(\lambda_{k}\right)
$$

Thus

$$
A\left(\lambda_{k}\right):=U\left(\lambda_{k}\right)^{-1}\left(\tilde{\Delta}_{j k k}+\lambda_{k}{X_{0 k j}}_{)} U\left(\lambda_{k}\right) \mid N\left(\lambda_{k}^{*}\right)\right.
$$

is $\left(H_{0 k k}\right)$ self-adjoint on $N\left(\lambda_{k}^{*}\right)$ and is holomorphic at $\lambda_{k}^{*}$, and its eigenvalue $\lambda_{j}\left(\lambda_{k}\right)$ is therefore real and holomorphic at $\lambda_{k}^{*}$.

In summary, the $\lambda\left(\lambda_{k}\right)$ and $x_{m}\left(\lambda_{k}\right)$ of (3.2) can be taken holomorphic at $\lambda_{k}^{*}$, and, since $\mu\left(\lambda_{k}\right)$ is nonconstant [5, Corollary 2.4$]$,

$$
\mu\left(\lambda_{k}^{*}\right)=\mu^{\prime}\left(\lambda_{k}^{*}\right)=\cdots=\mu^{(v-1)}\left(\lambda_{k}^{*}\right)=0 \neq \mu^{(v)}\left(\lambda_{k}^{*}\right)
$$

for some finite $v$. We are now ready for the construction of root vectors.

Theorem 3.1. In the simple case satisfying (3.2) and (3.5), the joint root subspace $J\left(\lambda^{*}\right)$; $=\bigcap_{n=1}^{k} N\left(\Gamma_{n}-\lambda_{n}^{*} I\right)^{d}, d=\operatorname{dim} F$, has a basis consisting of elements

$$
y_{j}=\sum_{i_{1}+\cdots+i_{k}=j} y_{1}^{i_{1}} \otimes \cdots \otimes y_{k}^{i_{k}}, \quad 0 \leqq j<v
$$

where

$$
W_{m}\left(\lambda^{*}\right) y_{m}^{l}=\sum_{n=1}^{k} V_{m n} \sum_{i=0}^{l-1} \gamma_{n}^{l-i} y_{m}^{i}, \quad 1 \leqq l<v
$$

$\gamma_{n}^{i}=\lambda_{n}^{(i)}\left(\lambda_{k}^{*}\right) / i !$ and $y_{m}^{0}=x_{m}$ as in $(*), 1 \leqq m, n \leqq k$.

Proof. By simplicity and [5, Theorem 3.3], $J\left(\lambda^{*}\right)$ is contained in $N\left(\Gamma_{k}-\lambda_{k}^{*} I\right)^{v}$ which has a basis $B=\left\{x^{\otimes}\left(\lambda_{k}^{*}\right), x^{\otimes \prime}\left(\lambda_{k}^{*}\right), \ldots, x^{\otimes(v-1)}\left(\lambda_{k}^{*}\right)\right\}$. Moreover

$$
\left(\Gamma_{n}\left(\mu\left(\lambda_{k}\right)\right)-\lambda_{n}\left(\lambda_{k}\right)\right) x^{\otimes}\left(\lambda_{k}\right)=0, \quad n=1, \ldots, k
$$

and repeated differentiation, together with (3.5), gives

$$
\left(\Gamma_{n}-\lambda_{n}^{*} I\right) x^{\otimes(l)}\left(\lambda_{k}^{*}\right)=\sum_{i=0}^{l-1} l ! \gamma_{n}^{l-i} x^{\otimes(i)}\left(\lambda_{k}^{*}\right) / i !, \quad 0 \leqq l<v .
$$


It follows inductively that

$$
\left(\Gamma_{n}-\lambda_{n}^{*} I\right)^{l} x^{\otimes(l-1)}\left(\lambda_{k}^{*}\right)=0
$$

so $J\left(\lambda^{*}\right)$ contains $B$, which is therefore a basis as required.

Thus it suffices to prove that

$$
y_{m}^{j}=x_{m}^{(j)}\left(\lambda_{k}^{*}\right) / j !, \quad 0 \leqq j<v,
$$

satisfy (3.6). This is clear for $j=0$, so assume $v>1$. Since $x_{m}=x_{m}\left(\lambda_{k}\right)$ is holomorphic at $\lambda_{k}^{*}$, we have by repeated differentiation of $(*)$

$$
W_{m}\left(\lambda^{*}\right) x_{m}^{(l)}\left(\lambda_{k}^{*}\right)=\sum_{i=0}^{l-1}(l !) \gamma_{n}^{l-i} V_{m n} x_{m}^{(i)}\left(\lambda_{k}^{*}\right) / i !
$$

for $1 \leqq m \leqq k$, and also for $m=k$ by virtue of (3.5).

Finally we compute

$$
y_{j}=x^{\otimes(j)}\left(\lambda_{k}^{*}\right) / j !=\sum_{i_{1}+\cdots+i_{k}=j} y_{1}^{i_{1}} \otimes \cdots \otimes y_{k}^{i_{k}}
$$

and (3.6) is established.

Remark 3.2. (1.6) is the special case of (3.6) obtained by setting $\lambda_{1}=\lambda_{2}=\cdots=\lambda_{k}$ and $y_{m}^{j}=x_{m}^{j}$.

Remark 3.3. Evidently (*) yields

$$
W_{m}\left(\lambda^{*}\right)^{\dagger} x^{\otimes}=0
$$

and repeated differentiation leads to

$$
\begin{aligned}
W_{m}\left(\lambda^{*}\right)^{\dagger} x^{\otimes(l)}\left(\lambda_{k}^{*}\right) / l ! & =\sum_{n=1}^{k} V_{m n}^{+} \sum_{i=0}^{l-1} \gamma_{n}^{l-i} x^{\otimes(i)}\left(\lambda_{k}^{*}\right) / i ! \\
& =\sum_{n=1}^{k} V_{m n}^{\dagger} z_{n},
\end{aligned}
$$

say. Thus our basis elements automatically satisfy equations of the form (1.5).

We return now to the general case, when $\operatorname{dim} N\left(\Gamma_{k}-\lambda_{k}^{*} I\right)$ is an arbitrary finite number. Geometrically, (3.2) generates $n_{c}$ curves parameterized by $\lambda\left(\lambda_{k}\right)$ and touching each of the $n_{k}:=\operatorname{dim} N\left(W_{k}\left(\lambda^{*}\right)\right)$ surfaces corresponding to the $k$ th equation of $(*)$. Each of the $n_{c} n_{k}$ possible combinations leads to a different set of vectors satisfying (3.6), each with its own initial element $y_{0}$ and its own length $v$. These $n_{c} n_{k}$ sets form our basis of $J\left(\lambda^{*}\right)$. 
Theorem 3.4. If $\lambda \in \mathbb{R}^{k}$ then $J\left(\lambda^{*}\right)$ is a direct sum of subspaces spanned by sets of root vectors $y_{j}$ as in Theorem 3.1 where the various initial elements $y_{0}=x^{\otimes}$ form a basis for $\bigcap_{n=1}^{k} N\left(\Gamma_{n}-\lambda_{n}^{*} I\right)=\bigotimes_{m=1}^{k} N\left(W_{m}\left(\lambda^{*}\right)\right)$.

Proof. $N\left(\lambda_{k}\right)$, defined as in (3.1), is now finite dimensional, so several branches $\left(\mu\left(\lambda_{k}\right), x\left(\lambda_{k}\right)\right)$ may exist holomorphic at $\lambda_{k}^{*}$. By Theorem 2.3 , the $\Gamma_{n}\left(\mu\left(\lambda_{k}\right)\right)$ on each set of coincident branches continue to generate a common eigenvector basis of $N\left(\lambda_{k}\right)$, provided $\mu\left(\lambda_{k}\right)$ is small and nonzero. We may now repeat the analysis of the simple case, choosing basis elements $x\left(\lambda_{k}\right)$ to be decomposable and to satisfy (3.2) for some $\lambda\left(\lambda_{k}\right)$, which are again $\mathbb{R}^{k}$-valued and holomorphic at $\lambda_{k}^{*}$ by $\left(H_{0 k k}\right)$ self-adjointness and holomorphy of the operators $A\left(\lambda_{k}\right)$ defined as in (3.3). Thus the $W_{m}\left(\lambda\left(\lambda_{k}\right)\right)$ in (3.2) are $H_{m}$ self-adjoint and holomorphic, and so we may choose the $x_{m}\left(\lambda_{k}\right)$ to be holomorphic at $\lambda_{k}^{*}$.

We now apply Theorem 3.1 to each branch in turn. An easy extension of [5, Theorem 3.3] shows that the $x^{\otimes(l)}\left(\lambda^{*}\right)$ form a basis of $N\left(\Gamma_{k}-\lambda_{k}^{*} I\right)^{d}$. Repeating the argument with $k$ replaced by each $n$ in turn, we automatically restrict the $y_{0}$ to $\bigcap_{n=1}^{k} N\left(\Gamma_{n}-\lambda_{n}^{*} I\right)$ and the $y_{j}$ generate a basis of $J\left(\lambda^{*}\right)$ as required.

\section{Remarks and special cases}

4.1. Determination of $\lambda_{n}^{i}$. At first sight this seems to require the eigenvalues $\lambda_{n}$ as functions of $\lambda_{k}$, but in fact much less information is needed. Let us illustrate for small $v$, using lower case letters for quadratic forms, e.g. $v_{m n}(x)=\left(x, V_{m n} x\right), \delta_{0 k k}(y)=\left(y, \Delta_{0 k k} y\right)$.

From (3.8) with $l=1$ we have, with $x_{m}=x_{m}\left(\lambda_{k}^{*}\right)$,

$$
0=\left(x_{m}, W_{m}\left(\lambda^{*}\right) x_{m}^{\prime}\left(\lambda_{k}^{*}\right)\right)=\sum_{n=1}^{k} \lambda_{n}^{\prime}\left(\lambda_{k}^{*}\right) v_{m n}\left(x_{m}\right), \quad 1 \leqq m<k .
$$

Since $\Delta_{0 k k} \gg 0$, we thus have a uniquely soluble system of linear equations in the unknowns $\lambda_{n}^{\prime}\left(\lambda_{k}^{*}\right), 1 \leqq n<k$. In fact

$$
\lambda_{n}^{\prime}\left(\lambda_{k}^{*}\right)=\delta_{0 k n}\left(x^{\otimes}\right) / \delta_{0 k k}\left(x^{\otimes}\right)
$$

i.e. a quotient of $(k-1) \times(k-1)$ determinants with entries of the form $v_{m n}\left(x_{m}\right)$, and no explicit differentiation is required to calculate $\gamma_{n}^{1}$.

We now use (3.8) to find $x_{m}^{\prime}\left(\lambda_{k}^{*}\right)$, again without explicit differentiation and proceed to $l$ $=2$, giving

$$
\begin{aligned}
0 & =\left(x_{m}, W_{m}\left(\lambda^{*}\right) x_{m}^{\prime \prime}\left(\lambda_{k}^{*}\right)\right) \\
& =2 \sum_{n=1}^{k} \lambda_{n}^{\prime}\left(\lambda_{k}^{*}\right)\left(x_{m}, V_{m n} x_{m}\left(\lambda_{k}^{*}\right)\right)+\sum_{n=1}^{k} \lambda_{n}^{\prime \prime}\left(\lambda_{k}^{*}\right) v_{m n}\left(x_{m}\right)
\end{aligned}
$$

which may be solved uniquely for $\lambda_{n}^{\prime \prime}\left(\lambda_{k}^{*}\right), 1 \leqq n<k$. This yields $\gamma_{n}^{2}$, and so on.

4.2. Jordan structure of the $\Gamma_{n}$. In the simple case, (3.7) shows that the $x^{\otimes(i)} / i$ ! form a Jordan basis for $\Gamma_{k}$, i.e. $\Gamma_{k}$ has Jordan block structure relative to this basis. Similarly $\Gamma_{n}$ 
has Toeplitz structure. Since any set of matrices commuting with a Jordan block will be of this form, the $\Gamma_{n}$ thus inherit no special properties (other than commutativity) from the multiparameter connection in the simple case. In the general case, however, the $\Gamma_{n}$ are direct sums of blocks as above, and this is a considerable specialization from the arbitrary commuting case.

4.3. Nonnegative $\Delta_{n}$. If at least one of the $\Delta_{n}$ is nonnegative definite, say $\Delta_{k} \geqq 0$, then $\lambda_{k}$ must be real [7, Lemma 5.1]. Thus (3.3) gives

$$
\lambda_{j}=\delta_{0 k k}\left(x^{\otimes}\right)^{-1}\left(\lambda_{k} \delta_{0 k j}\left(x^{\otimes}\right)+\delta_{j k k}\left(x^{\otimes}\right)\right)
$$

in the quadratic form notation of 4.1 , and so $\lambda \in \mathbb{R}^{k}$.

If $\Delta_{k} \gg 0$ then $\lambda_{k}$ is an eigenvalue of the compact self-adjoint operator $B_{k}=\Delta_{k}^{-1} \Delta_{0}$ on $D\left(\Delta_{k}^{1 / 2}\right)$ with inner product given by $[x, y]=\left(\Delta_{k}^{1 / 2} x, \Delta_{k}^{1 / 2} y\right)$, and is thus a nondefective eigenvalue. The analysis of 4.2 thus shows that one may take $v=1$ in (1.4). This case occurs e.g. when $T_{m} \gg 0$, i.e. ULD.

If $\Delta_{k} \geqq 0$ but not $\gg 0$, i.e. $N\left(\Delta_{k}\right)$ is nontrivial, then $\Gamma_{k}$ has Jordan chains of length at most two, and if the length is two then $\lambda_{k}=0$ [7, Lemma 5.1]. Appealing again to 4.2, then, we see that $F$ is spanned by Jordan chains of the form $\left\{x^{\otimes}\right\}$ or $\left\{x^{\otimes}, x^{\otimes^{\prime}}\right\}$.

The analysis of 4.1 thus gives a complete description of $F$ in terms of the original data in $(*): x^{\otimes}=x_{1} \otimes \cdots \otimes x_{k}, x_{m}$ as in $(*)$ and

$$
x^{\otimes \prime}=\sum_{m=1}^{k} x_{1} \otimes \cdots \otimes x_{m-1} \otimes x_{m}^{\prime} \otimes x_{m+1} \otimes \cdots \otimes x_{k}
$$

where

$$
W_{m}(\lambda) x_{m}^{\prime}=\sum_{n=1}^{k} \delta_{0 k n}\left(x^{\otimes}\right) V_{m n} x_{m} / \delta_{0 k k}\left(x^{\otimes}\right)
$$

In the case of $k=2$ Sturm-Liouville equations, this result can be obtained from [9, Theorem 5.5] although it is stated differently. In the case where each $T_{m} \geqq 0$ (so each $\Delta_{n} \geqq 0$ ) the Jordan chain structure of $F$ (and its dimension) were analysed in [7, Section 5] but without explicit formulae for $x^{\otimes}$.

4.4 An example. Let $k=2, H_{1}=H_{2}=\mathbb{C}^{2}$,

$$
T_{1}=T_{2}=\left[\begin{array}{ll}
1 & 0 \\
0 & 0
\end{array}\right], \quad V_{11}=\left[\begin{array}{ll}
2 & 1 \\
1 & 2
\end{array}\right], \quad V_{21}=\left[\begin{array}{rr}
-4 & 1 \\
1 & -4
\end{array}\right], \quad V_{22}=-2 V_{12}=\left[\begin{array}{ll}
2 & 0 \\
0 & 2
\end{array}\right] .
$$

Then $0=\operatorname{det} W_{1}(\lambda)=\varepsilon^{2}+\varepsilon-\lambda_{1}^{2}, \varepsilon=2 \lambda_{1}-\lambda_{2}$ and $0=\operatorname{det} W_{2}(\lambda)=4 \varepsilon^{2}-2 \varepsilon-\lambda_{1}^{2}$. The solutions are $\varepsilon=1$, giving $\lambda=( \pm \sqrt{ } 2, \pm 2 \sqrt{ } 2-1)$, and $\varepsilon=0$, giving $\lambda=0$ (a double root). When $\varepsilon=1$, we calculate eigenvectors

$$
x_{1}=\left[\begin{array}{c}
\mp 1 \\
\sqrt{ } 2
\end{array}\right] \text { and } x_{2}=\left[\begin{array}{c} 
\pm \sqrt{ } 2 \\
1
\end{array}\right] .
$$


When

$$
\varepsilon=0,\left[\begin{array}{ll}
1 & 0 \\
0 & 0
\end{array}\right] x_{m}=\left[\begin{array}{l}
0 \\
0
\end{array}\right], \quad \text { say } x_{m}=\left[\begin{array}{l}
0 \\
1
\end{array}\right], m=1,2 .
$$

The root vector $x^{\otimes \prime}=x_{1}^{\prime} \otimes x_{2}+x_{1} \otimes x_{2}^{\prime}$ may be calculated via (4.1). Evidently

$$
\delta_{021}\left(x^{\otimes}\right)=-v_{12}\left(x_{1}\right)=1 \quad \delta_{022}\left(x^{\otimes}\right)=v_{11}\left(x_{1}\right)=2
$$

so

$$
\left[\begin{array}{ll}
1 & 0 \\
0 & 0
\end{array}\right] x_{1}^{\prime}=1 / 2\left[\begin{array}{l}
1 \\
2
\end{array}\right]+1\left[\begin{array}{r}
0 \\
-1
\end{array}\right]=\left[\begin{array}{c}
1 / 2 \\
0
\end{array}\right], \quad \text { say } x_{1}^{\prime}=\left[\begin{array}{c}
1 / 2 \\
0
\end{array}\right]
$$

and

$$
\left[\begin{array}{ll}
1 & 0 \\
0 & 0
\end{array}\right] x_{2}^{\prime}=1 / 2\left[\begin{array}{r}
1 \\
-4
\end{array}\right]+1\left[\begin{array}{l}
0 \\
2
\end{array}\right]=\left[\begin{array}{c}
1 / 2 \\
0
\end{array}\right], \quad \text { say } x_{2}^{\prime}=\left[\begin{array}{c}
1 / 2 \\
0
\end{array}\right]
$$

Using the isomorphism $H^{2} \cong \mathbb{C}^{4}$, we may write the two eigentensors corresponding to $\varepsilon=1$ as $(-\sqrt{ } 2, \mp 1, \pm 2, \sqrt{ } 2)$, the one corresponding to $\varepsilon=0$ as $(0,0,0,1)$ and the root vector $x^{\otimes \prime}$ as $(0,1 / 2,0,0)+(0,0,1 / 2,0)=(0,1 / 2,1 / 2,0)$. It is readily verified that these four elements are indeed a basis of $\mathbb{C}^{4}$.

Acknowledgement. I thank the Universities of Dundee and Strathclyde for their hospitality during the preparation of this work. I also thank Patrick Browne for various helpful discussions and for criticizing the original draft.

\section{REFERENCES}

1. F. V. Atkinson, Multiparameter spectral theory, Bull. Amer. Math. Soc. 74 (1968), 1-27.

2. F. V. AtKinson, Multiparameter Eigenvalue Problems Vol. 1 (Academic Press, 1972).

3. P. A. Binding, Multiparameter definiteness conditions, Proc. Roy. Soc. Edinburgh 89A (1981), 319-332.

4. P. A. Binding, Left definite multiparameter eigenvalue problems, Trans. Amer. Math. Soc. 272 (1982), 476-486.

5. P. A. Binding and P. J. Browne, Applications of two parameter spectral theory to symmetric generalised eigenvalue problems, preprint.

6. P. A. Binding and K. Seddighi, On root vectors for self-adjoint pencils, J. Funct. Anal. 70 (1987), 117-125.

7. P. A. Binding and K. Seddighi, Elliptic multiparameter eigenvalue problems, Proc. Edinburgh Math. Soc. 30 (1987), 215-228.

8. M. Faierman, Expansions in eigenfunctions of two-parameter system of differential equations I-IV, preprints. 
9. G. A. Gadzhiev, On a multitime equation and its reduction to a multiparameter spectral problem, Soviet Math. Dokl. 32 (1985), 710-713.

10. G. A. IsaEv, On root elements of multiparameter spectral problems, Soviet Math. Dokl. 21 (1980), 127-130.

11. A. Kallstrom and B. D. Sleeman, Solvability of a linear operator system, J. Math. Anal. Appl. 55 (1976) 785-793.

12. A. Kallstrom and B. D. Sleeman, A left definite multiparameter eigenvalue problem in -ordinary differential equations, Proc. Roy. Soc. Edinburgh 74A (1976), 145-155.

13. T. Kato, Perturbation Theory for Linear Operators (Springer-Verlag, 1976).

Department of Mathematics and Statistics

UNIVERSITY OF CALGary

Calgary, Alberta

CANADA T2N IN4 